\title{
1-S05-3
}

\section{Pharmacological analysis of the regulatory mechanisms of sperm hyperactivation by ouabain}

\section{Gen L. Takei}

Dept. Pharmacol. Toxicol. Dokkyo Med. Univ.

Mammalian sperm activate their motility upon ejaculation, but sperm motility needs further change to whiplash-like motility to penetrate and fertilize the egg. This specialized motility is called "hyperactivation". It was reported that extracellular $\mathrm{Na}^{+}$is involved in regulation of hyperactivation. $\mathrm{Na}^{+} / \mathrm{K}^{+}$ATPase (NKA) plays a major role in the regulation of cellular $\mathrm{Na}^{+}$homeostasis. The catalytic subunit of NKA is an $\alpha$ subunit, and there are $\alpha 1$ and $\alpha 4$ subunits in sperm. The sensitivity of these two $\alpha$ subunits to ouabain, a specific NKA inhibitor, is quite different, that is, $10^{-6} \mathrm{M}$ ouabain inhibits NKA $\alpha 4$ while $>10^{-5} \mathrm{M}$ ouabain inhibits both the $\alpha 1$ and $\alpha 4$. Therefore, this difference of sensitivity enables us to distinguish the physiological role of NKA $\alpha$ subunits on sperm function by pharmacological approach. In this symposium, I will introduce my latest study which showed, by utilizing this pharmacological property of ouabain, that the NKA $\alpha 1$ subunits is necessary for the maintenance of motility while the $\alpha 4$ subunit is necessary for the hyperactivation-associated change in flagellar movement. These results suggest that NKA $\alpha 4$ subunit is an attractive target for the male contraception. 